\title{
Searches with multilepton final states
}

\author{
Celso MARTINEZ RIVERO ${ }^{1,2}$ \\ ${ }^{1}$ IFCA (CSIC-UC). Avda. Los Castros s/n 39005 Santander (Spain). \\ ${ }^{2}$ On behalf of ATLAS and CMS collaborations
}

\begin{abstract}
In this talk, the latest results from CMS and ATLAS on searches for new physics with multiple leptons in the final state are presented using up to $20 / \mathrm{fb}$ of data from the $8 \mathrm{TeV}$ LHC run of 2012. Interpretation of results in terms of SUSY searches for production of gauginos and sleptons are shown. For RP conserving models, both neutralinos and gravitinos as lightest supersymmetric particles are considered.
\end{abstract}

\section{Introduction}

Many searches for supersymmetry (SUSY) carried out at the CERN Large Hadron Collider (LHC) have focused on models with cross sections dominated by the production of strongly interacting new particles. The corresponding final states exhibit a high level of hadronic activity.

In contrast, in this presentation, we describe searches motivated by the direct electroweak production of charginos, neutralinos, and sleptons. The corresponding final states do not necessarily contain much hadronic activity and thus may have eluded detection in other searches. This signature characterizes SUSY models that describe the pair production of charginos and neutralinos, mixtures of the SUSY partners of the gauge and Higgs bosons, and of sleptons, the SUSY partners of leptons. Depending on the mass spectrum, the charginos and neutralinos can have significant decay branching fractions to leptons or on-shell vector bosons, yielding multilepton final states. Similarly, slepton pair production gives rise to final states with leptons. In all these cases, two stable, lightest-SUSY-particle (LSP) dark matter candidates are produced, which escape without detection, leading to significant missing transverse energy. Neutrinos present in the final state yield additional $E_{T} m i s s$. We search also multiple new regions of the supersymmetric parameter space not yet excluded by previous multilepton searches of LHC data at a centre of mass energy of $8 \mathrm{TeV}$. As the search is not tailored to any particular SUSY scenario, its results can be used to constrain a broad range of relevant SUSY models.

Finally, R-Parity violation is included presented in terms of the visible cross-section for new physics processes with 4 lepton signature, interpreted in a variety of Rparity conserving and violating simplified models and general gauge mediated models.

We will structure this presentation in three main lines:

-Electroweakino production with a luminosity of $20.7 \mathrm{fb}-1$ for ATLAS and $9.2 \mathrm{fb}-1$ for CMS

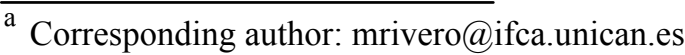

- General multilepton with a luminosity of $20.7 \mathrm{fb}-1$ for ATLAS and $19.5 \mathrm{fb}-1$ for CMS

-RPV with or without stop with a luminosity of 20.7 fb-1 for ATLAS and $19.5 \mathrm{fb}-1$ for CMS

\section{Electroweakino production}

We search here for four different scenarios with two leptons at the end of the analysis:

Direct-slepton scenario The direct-slepton models are based on the pMSSM. The masses of all charginos and neutralinos (apart from the LSP) are set to $2.5 \mathrm{TeV}$. The sensitivity of the present search is determined for models with varying slepton and LSP masses. The common selectron and smuon mass is generated in the range 90$370 \mathrm{GeV}$. The cross-section for direct slepton pair production (per slepton flavour) in these models decreases from 127 to $0.5 \mathrm{fb}$ for left-handed sleptons, and from 49 to $0.2 \mathrm{fb}$ for right-handed sleptons, as the slepton mass increases from 90 to $370 \mathrm{GeV}$.

Chargino-to-slepton scenario The chargino-to-slepton decays are simulated in simplified models, in the scenario of charginos decaying into the lightest neutralino via an intermediate on-shell charged slepton, in which the masses of neutralino, slepton, sneutrino and chargino are the only free parameters. The squarks are assumed to be well beyond the kinematical reach. The charginos are pair-produced via the s-channel exchange of a virtual gauge boson and decay via left-handed sleptons. The cross-section for chargino pair production calculated under the assumption that the chargino is $95 \%$ wino-like (with a small higgsino component), is as high as $5 \mathrm{pb}$ for a chargino mass of $100 \mathrm{GeV}$ and decreases rapidly at higher masses, reaching $9 \mathrm{fb}$ at $450 \mathrm{GeV}$.

Chargino-to-W scenario Simulated samples for chargino-to-W decays, where the sleptons are mass 
decoupled, were also produced with simplified models. The mass grid spans the chargino vs neutralino plane in $10-\mathrm{GeV}$ steps on both axes. The cross-section used for the chargino pair production is the same as for the chargino-to-slepton scenario. The branching ratio is assumed to be $100 \%$.

GMSB model An additional simplified model sample is generated to probe the GMSB model. In this model, the LSP is the gravitino G, the NLSP is the chargino with mass equal to $110 \mathrm{GeV}$, and in addition there are two other light neutralinos with masses $113 \mathrm{GeV}$ and 130 $\mathrm{GeV}$. All coloured sparticle are assumed to be very heavy. Because of the small mass differences, the decay products of the off-shell $W$ and $Z$ bosons are unlikely to be detected. As a result, all of the four production channels result in the same experimental signature, and their production cross-sections can be added together for the purpose of this search. Leptonic decays of the two onshell $W$ bosons produce the same final-state as in the chargino-to-W scenario above.

\subsection{Direct-slepton scenario}

After some preliminary cuts, a stransverse mass is used defined as $\mathrm{m}_{\mathrm{T} 2}$ for ATLAS or $\mathrm{M}_{\mathrm{CT} \text {,Perp }}$ for CMS which gives a kinematical endpoint at $\mathrm{W}$ mass. Figure 1 shows the distribution of $m_{T 2}$ requiring missing energy bigger then $40 \mathrm{GeV}$ and the $Z$ veto. Good agreement between data and Monte Carlo is observed for all variables and samples.

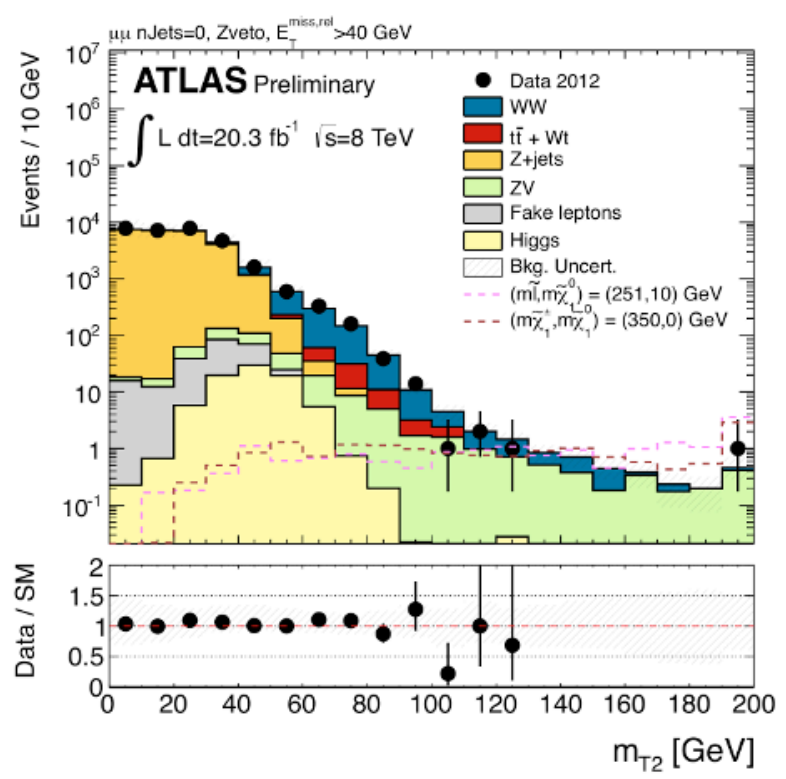

Figure 1. Distributions of $m_{T 2}$ in the $\mu+\mu-$ event samples satisfying $E_{T}$ miss, rel $>40 \mathrm{GeV}$, and the $Z$ veto. The expected distributions from the $W W, t t^{-}$and $Z V$ processes are corrected with data- driven scale factors. The hashed regions represent the total uncertainties on the background estimates. The right-most bin of each plot includes overflow. Illustrative SUSY benchmark models are super-imposed.
The $95 \% \mathrm{CL}$ are shown in figure 2 for the direct productions of left and right-handed slepton production. The plot shows the 95\% CLs expected (dashed black) and observed (solid red) limits, including all uncertainties except the theoretical uncertainty on the signal cross section. The solid yellow band indicates the impact of experimental uncertainties on the expected limits whereas the dashed red lines around the observed limit show the changes in the observed limit as the signal cross-sections are scaled up and down by the $1 \sigma$ theoretical uncertainties. A common value for left and right-handed selectron and smuon mass between $90 \mathrm{GeV}$ and $320 \mathrm{GeV}$ is excluded for a massless neutralino, where these numbers correspond to the observed value minus the signal theoretical uncertainty.

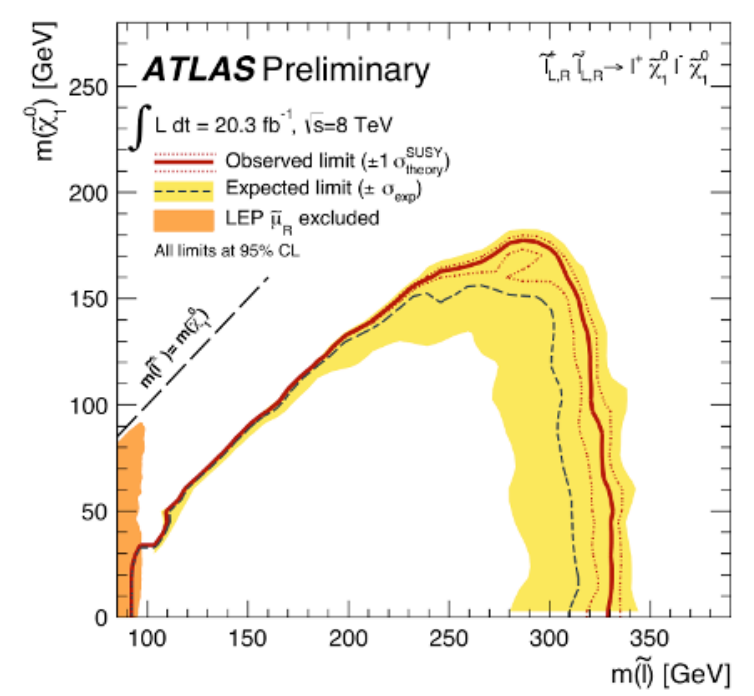

Figure 2. $95 \% \mathrm{CL}$ exclusion limits for both right and lefthanded (mass degenerate) selectron and smuon production in the mass of neutralino and mass of the slepton plane. The dashed and solid lines show the 95\% CLs expected and observed limits, respectively, including all uncertainties except for the theoretical signal cross-section uncertainty (PDF and scale). The solid band around the expected limit shows the $\pm 1 \sigma$ result where all uncertainties, except those on the signal crosssections, are considered.

\subsection{Chargino-to-slepton scenario}

The Feynmann diagram corresponds to Figure 3.

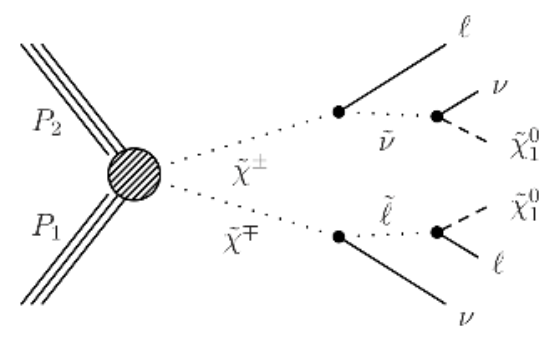

Figure 3. Chargino-pair production leading to opposite-sign lepton pairs. 
In this case Figure 4 shows limits on the chargino pairproduction cross section times branching ratio for the processes of Fig. 3. The limits for chargino-pair production are set using both the opposite and sameflavor channels.

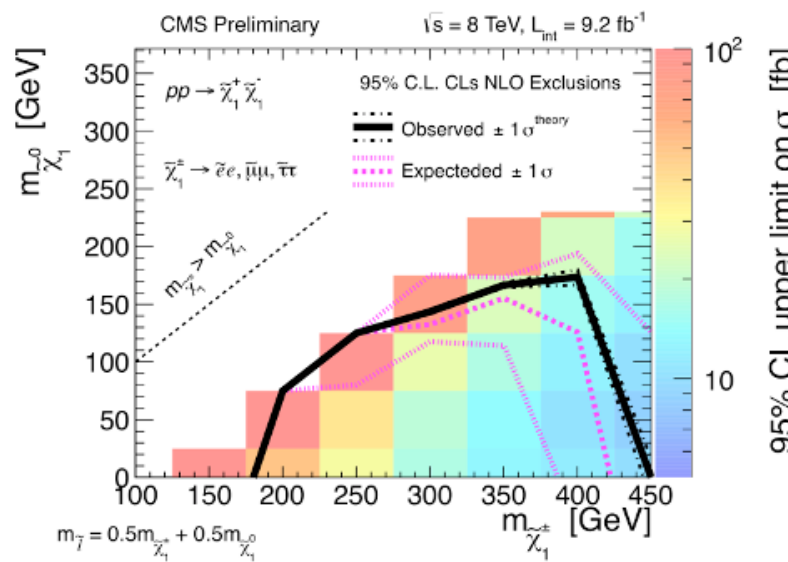

Figure 4. Limits on chargino pair production cross section times branching ratio for charginos and sleptons decaying as in Fig. 3.

\subsection{Chargino-to-W scenario}

If one takes only electron and muon leptons in the event, and $100 \% \mathrm{BR}$ in the channel chargino to $\mathrm{W}$ scenario, one can explore the heavy slepton scenario in which sleptons are too heavy to be produced. Three different signal regions are created in order to look for this kind of Opposite Sign emu samples. For each signal region, a simultaneous likelihood fit to the signal regions and the control regions is performed to normalise the top, $W W$ and $Z V$ background estimates and to determine or limit a potential signal contribution. Figure 5 show the comparison between data and the $\mathrm{SM}$ prediction for $\mathrm{m}_{\mathrm{T} 2}$ variable in different signal regions.

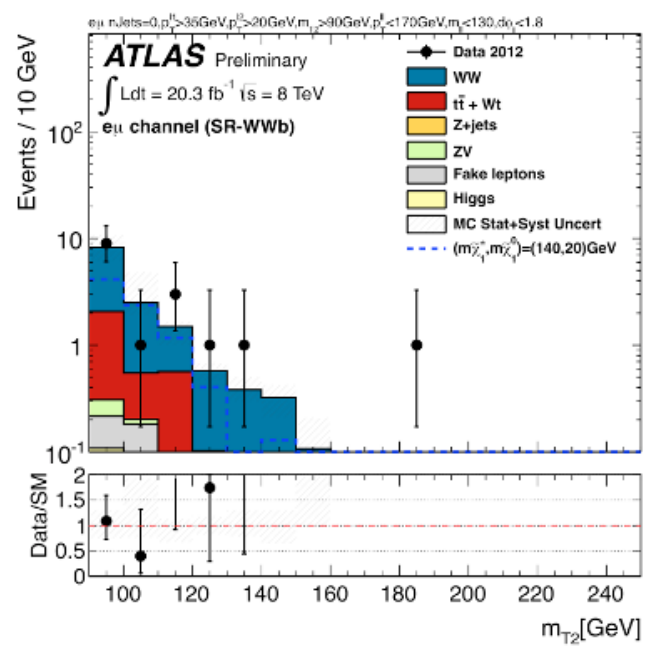

Figure 5. Distribution of $\mathrm{m}_{\mathrm{T} 2}$ in a signal region.
Figure 6 shows the observed and expected 95\% CLs upper limits on the cross section as a function of mass of the chargino for a massless neutralino normalised to the model cross-section. The excluded cross-section is above the model cross-section by a factor 1.9-2.8 in the range $100-190 \mathrm{GeV}$ and then degrades gradually to 4.7 when reaching a chargino mass of $250 \mathrm{GeV}$.

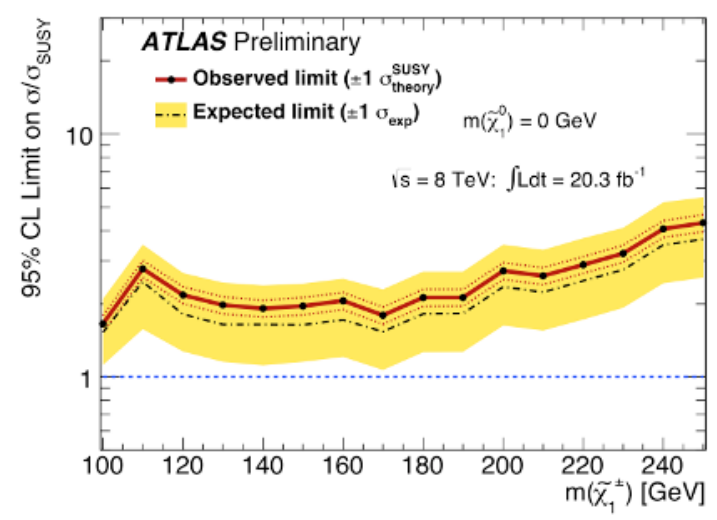

Figure 6. Observed and expected 95\% CLs upper limits of the cross-section as a function of mass of the chargino for massless neutralino normalised to the simplified-model cross-section.

\subsection{GMSB model.}

The CLs value is also calculated for the GMSB model point where the chargino is the NLSP. The observed CLs value is found to be 0.52 using the most sensitive signal region for this point. The expected and observed 95\% CL limit on o/oSUSY are 2.6 and 2.9 , respectively.

If one looks for three leptons at the end of the analysis, the following diagrams, like those in figure 7 or 8 , have a very low background. A 3D binning is made using the transverse mass, missing energy and the invariant mass of the two highest leptons in order to separate a possible signal from background.

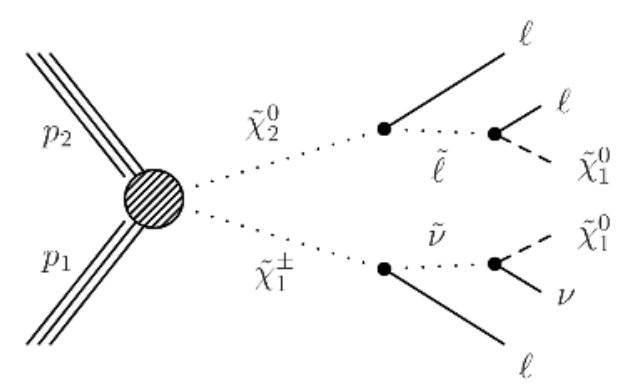

Figure 7. Chargino-neutralino production at LHC leading to a three-lepton final state

The principal backgrounds are from WZ production with three genuine isolated leptons that are "prompt" (created at the primary vertex), and from tt production with two 


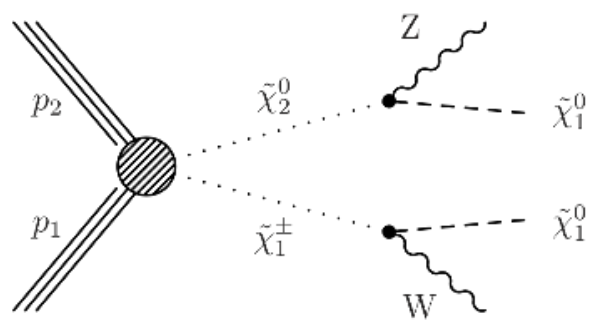

Figure 8. Chargino-neutralino production leading to on-shell W with missing energy.

genuine prompt leptons and a third non-prompt lepton misclassified as prompt. (see Figure 9)

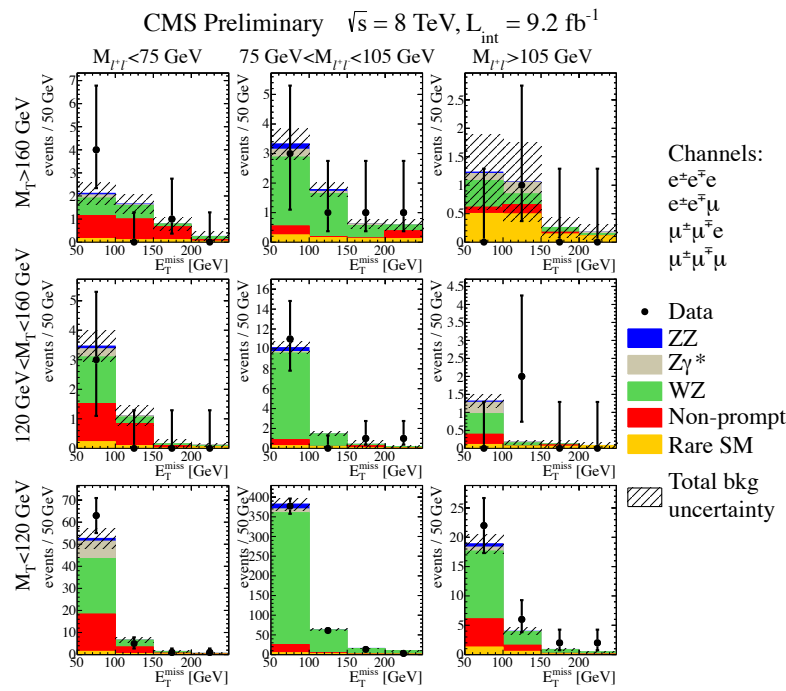

Figure 9. Observed $E_{T} m i s s$ distribution for three-lepton data events with an ee or $\mu \mu$ OSSF dilepton pair, where the third lepton is either an electron or a muon. SM expectations are also shown. "Rare SM" refers to standard model processes such as $\mathrm{ZZ}, \mathrm{ttZ}, \mathrm{ttW}$, and triboson production.

When comparing signal to background, one can extract the exclusion limits in Simplified SUSY models, either for channels like those in Figure 7 -chargino-neutralino to three lepton final state- as for channels like those in Figure 8 -chargino-neutralino leading to on-shell $\mathrm{W}$ and missing energy. Figure 10 shows the 95\%CL upper limit on the chargino-neutralino production NLO cross section times branching fraction in the flavor-democratic scenario, for the combined analysis of the three-lepton search and the same-sign dilepton search for CMS.

In Figure 11, the limit in the simplified model with charginos and neutralinos decaying via gauge bosons is shown.

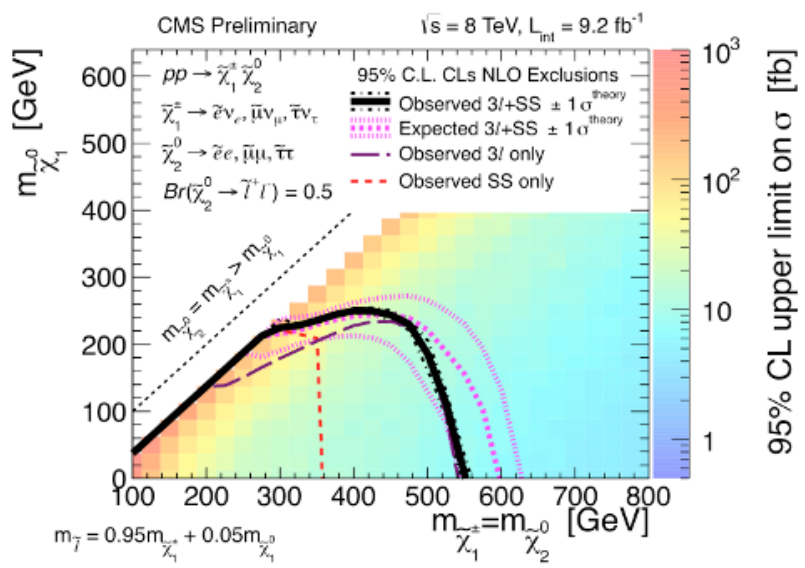

Figure 10. 95\% CL upper limit on chargino-neutralino production NLO cross section times branching fraction.

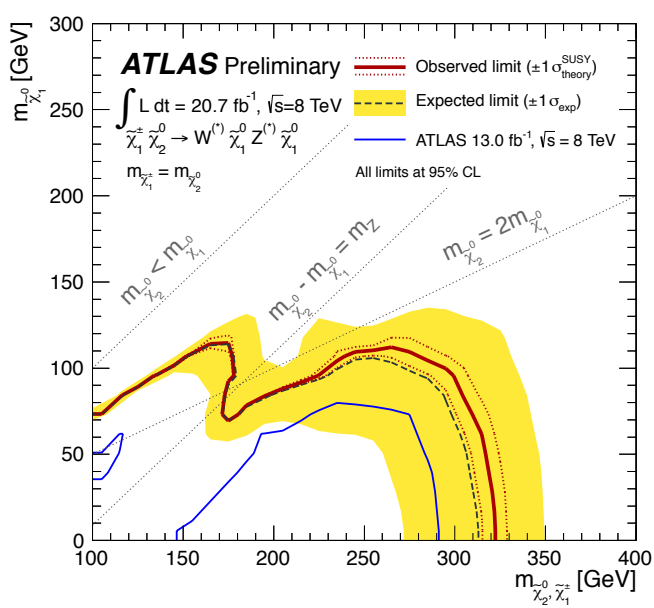

Figure 11. 95\% CLupper limit contours for chargino and neutralino production in the simplified model scenario decay via gauge bosons.

\section{General multilepton}

For SUSY models that predict the production of third generation scalar quarks (squarks), the addition of a b-jet requirement in the signature provides a powerful tool that has been exploited in several searches. SUSY scenarios leading to the production of light stop, sbottom, and gluino are well motivated theoretically and have only been partially explored by existing searches. The motivation arises from naturalness considerations related to protecting the Higgs mass against large loop corrections in the standard model (SM). The first- and second-generation squarks, in contrast, are not required to be light in such scenarios. Figure 12 shows an example of one of such scenarios: the production of a pair of gluinos, which decay to four top quarks and two neutralinos. 


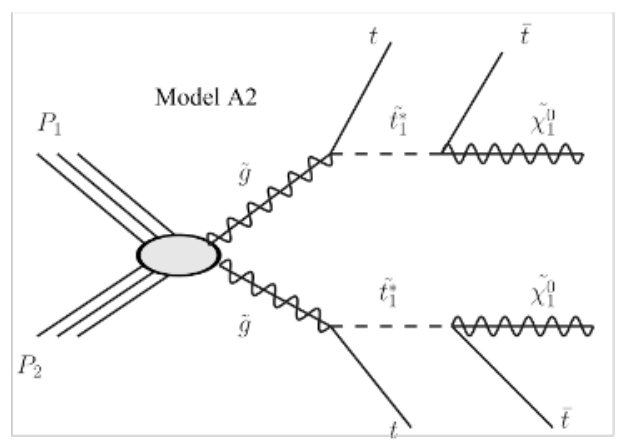

Figure 12. Gluino-pair production with subsequent decay to four top quarks and two lightest SUSY particles

In this case a bidimensional Plot of the $\mathrm{H}_{\mathrm{T}}$ vs de $\mathrm{E}_{\mathrm{T}}$ miss for off-shell $\mathrm{Z}$ are used. The high $\mathrm{H}_{\mathrm{T}}$ and high Njets are used for the signal. A data-driven prediction for nonprompt and misidentified leptons is used together with a requirement that the number of b-tagged jets is 2 or more. Figure 13 show the bidimensional plot in the plane $\mathrm{H}_{\mathrm{T}} \mathrm{vs}$ de $\mathrm{E}_{\mathrm{T}}$ miss, while Figure 14 shows the $95 \% \mathrm{CL}$ upper limits derived using the CLs method.

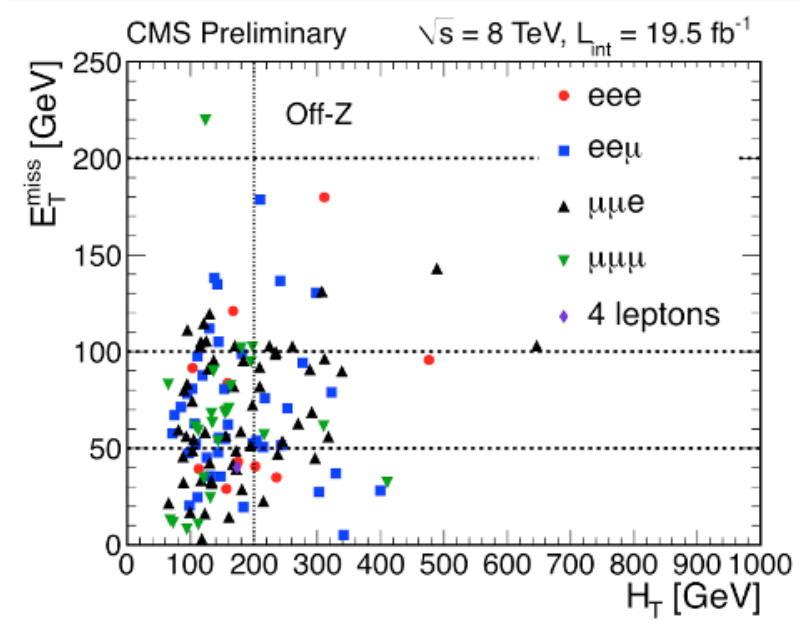

Figure 13. Distribution of events after the baseline event selection in data in the Emiss vs. $H_{T}$ plane for Off-Z categories. The requirement $E_{T}$ miss $>50 \mathrm{GeV}$ has not been applied to illustrate the background population at low $\mathrm{E}_{\mathrm{T}} \mathrm{miss}$.

On the other hand, a four lepton final state can be obtained asking for regions of high missing energy and high efficient mass (efficient mass is equal to the sum of the missing energy, and the transverse momemtun of electrons, muons, taus and jets). Figure 15 shows a plot with the missing energy for data at $8 \mathrm{TeV}$ from ATLAS, (without the cut of at least $50 \mathrm{GeV}$ for $\mathrm{E}_{\mathrm{T}}$ miss).

With this analysis, exclusion plots in different SUSY scenarios can be obtained. Figure 16 shows the 95\% CL exclusion limits for an scenario in which the difference in mass between the third neutralino and the second neutralino is zero.

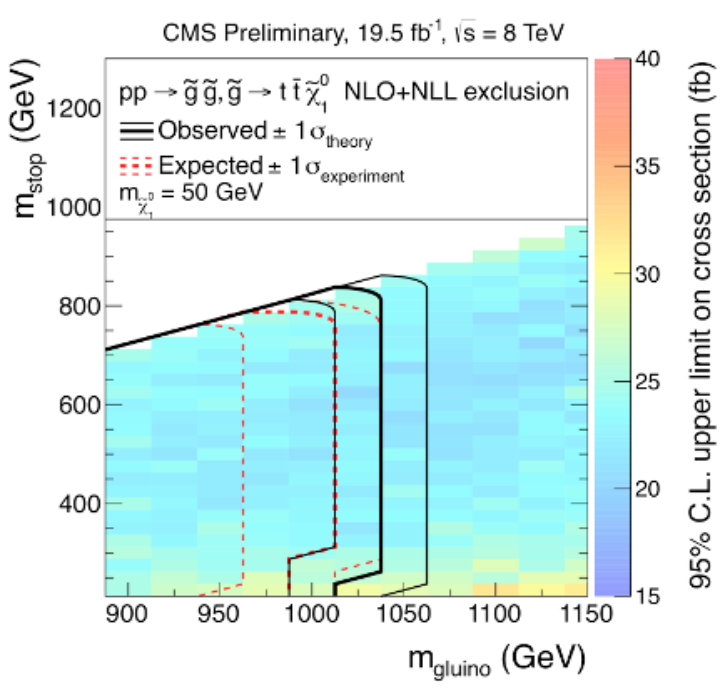

Figure 14. 95\% CL upper limits on the gluino pair production to 4 tops and two neutralinos.

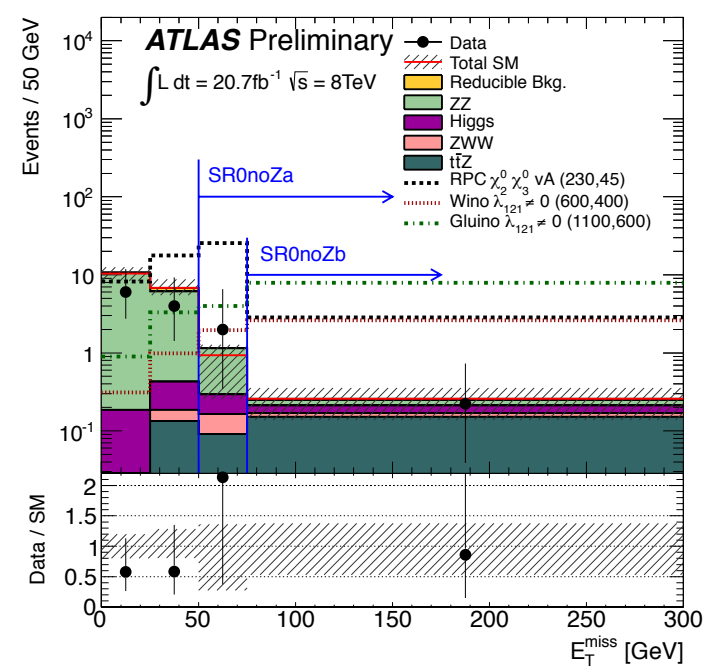

Figure 15 Distributions of the $E_{T} m i s s$ in events with $\geq 41 \geq 0 \tau$ and a $Z$ veto.

\section{R-Parity violation with or without stop.}

An interpretation can be done of the search results in the context of supersymmetric models with diminished missing transverse energy signatures arising from light stop pair production with $R$-parity violating decays of the lightest supersymmetric particle. Assigning R-parity fields as $R p=(-1)^{3 \mathrm{~B}+\mathrm{L}+2 \mathrm{~s}}$, where $B$ and $L$ are baryon and lepton numbers, and $s$ is the particle spin, all SM particle fields have $R p=+1$ while all superpartner fields have $R p$ $=-1$. The most general RPV renormalizable superpotential includes three trilinear $R p$ violating terms parametrized by the Yukawa couplings $\lambda_{i j k,} \lambda^{\prime}{ }_{i j k}$, and $\lambda^{\prime \prime}{ }_{i j k}$. 


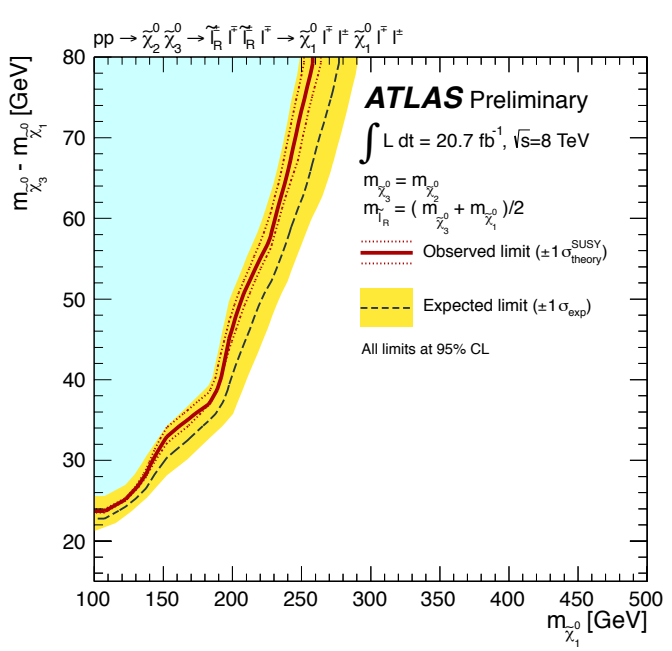

Figure 16 95\% CL exclusion limit for RPC simplified models for the direct production of second and third neutralinos.

If one define $\mathrm{S}_{\mathrm{T}}=\mathrm{E}_{\mathrm{T}}$ miss $+\mathrm{H}_{\mathrm{T}}+\mathrm{L}_{\mathrm{T}}$ (being $\mathrm{L}_{\mathrm{T}}$ the sum of the charged lepton $\mathrm{p}_{\mathrm{T}}$ ), figure 17 shows the $\mathrm{S}_{\mathrm{T}}$ distribution for the case of events with 3 leptons (without taus) in the off-Z scenario with at least $1 \mathrm{~b}$-tagged jet.

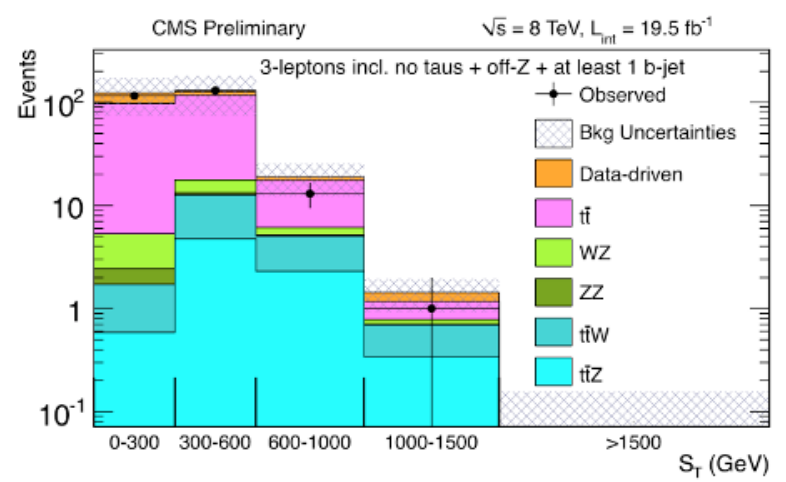

Figure $17 \mathrm{~S}_{\mathrm{T}}$ plot for events with three leptons and no hadronically decaying taus

Stop mass limits can be shown for these scenarios. In Figure 18 the RPV coupling $\lambda_{122}$ is shown, with the region to the left of the curve excluded.

Another analysis looks for four isolated leptons (electrons or muons) in LRPV neutralino decay containing at least one OSSF lepton pair (the only selection applied.). pMSSM covers MSSM spectra and allows to get very generic exclusion SUSY models with LRPV term. The search is done without tau contribution (due to the presence of LRPV terms $\lambda_{121}$ and $\lambda_{122}$ ). Figure 19 shows the generic mass exclusion on inclusive LRPV production.

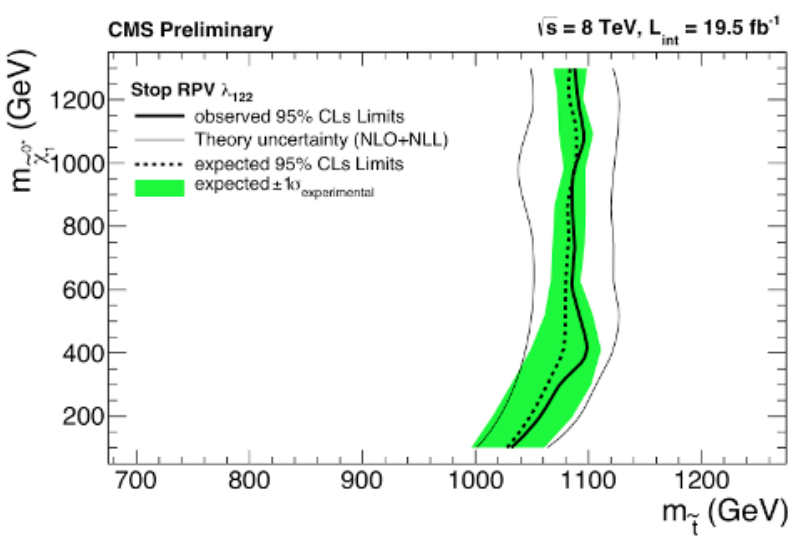

Figure 18 95\% CL limits for stop mass in models with RPV couplings $\lambda_{122}$

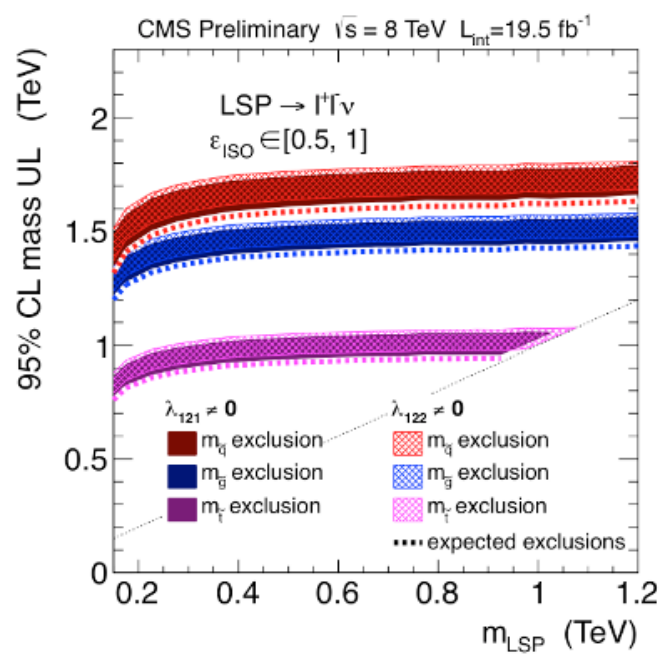

Figure 19 Mass exclusion for a generic RPV SUSY cross section limit.

\section{Conclusions:}

Many searches for SUSY in multilepton final states have been performed at $\mathrm{LHC}$ with data at $8 \mathrm{TeV}$; no excess beyond Standard Model expectation is observed. Stringent constraints on masses of charginos, neutralinos and sleptons are made. One should stay tuned for more results in the near future.

\section{References}

The following conference papers have been used:

- ATLAS-CONF-2013-049, 028, 035 and 036

- CMS-PAS-SUS-13-003, 08, 010 and

- CMS-PAS-SUS-12-022 and 026 